\title{
Pulmonary thromboembolism: a rare but life-threatening complication of nephrotic syndrome
}

Heeyeon Cho, MD, PhD

Department of Pediatrics, Samsung Medical Center, Sungkyunkwan University School of Medicine, Seoul, Korea

\section{Key message}

Pulmonary thromboembolism (PTE) is often diagnosed in children with nephrotic syndrome. Massive PTE can cause hemodynamic instability, right ventricular failure, and circulatory collapse. Extracorporeal membrane oxygenation can be used for massive PTE as a method of hemodynamic support and adjunct to surgical embolectomy.

Pulmonary thromboembolism (PTE) is often diagnosed in children with underlying medical disorders, and a meta-analysis reported that its prevalence among patients with nephrotic syndrome was 7.93\%. . $^{1}$ Patients with nephrotic syndrome have a hypercoagulable state caused by several factors, such as abnormalities in platelet activation (increased number and aggregation) and an imbalance between anticoagulation/antithrombosis (decreased antithrombin III levels) and procoagulant/ prothrombotic mechanisms (increased factor V, factor VII, and fibrinogen levels), leaving them at increased risk for deep venous and arterial thrombosis, renal vein thrombosis, and PTE. ${ }^{2,3)}$

Thromboembolic complications tend to present more severely, which may be associated with severe hypoalbuminemia in children with nephrotic syndrome. ${ }^{4)}$ Additionally, the thromboembolic risk is higher in adolescents with nephrotic syndrome than in children. In a report by You et al., ${ }^{5)}$ an adolescent had persistent proteinuria and hypoalbuminemia despite steroid therapy and was at risk for thromboembolism. However, prophylactic anticoagulation is not usually recommended because of a lack of large randomized trials and guidelines. ${ }^{2)}$

PTE symptoms include pleuritic chest pain, shortness of breath, and hemoptysis, and its diagnosis can be missed or delayed in children due to its nonspecific symptoms and difficulty assessing pain in young children. ${ }^{6}$ Thus, the physician's suspicion is highly necessary to diagnose PTE. ${ }^{6}$ In patients with nephrotic syndrome, a high plasma D-dimer level was associated with the occurrence of PTE. ${ }^{7)}$ Regarding underlying etiologic factors, it is possible that children with nephrotic syndrome and PTE may have other thrombophilia states such as anticardiolipin antibody, antithrombin deficiency, and protein $\mathrm{C}$ and $\mathrm{S}$ deficiencies, and it may be necessary to evaluate the presence of risk factors in addition to nephrotic syndrome. ${ }^{8)}$
The management guidelines in children are extrapolated from adult data, and treatment includes thrombolysis or thrombectomy and pharmacologic anticoagulation.8) Anticoagulation should be initiated with a rapid-acting agent, either unfractionated or low-molecular-weight heparin, followed by continued anticoagulation with low-molecular-weight heparin or a vitamin K antagonist. ${ }^{9,10)}$ Low-molecular-weight heparin is now widely used in children. There is no consensus regarding the duration of therapy, with the American College of Chest Physicians (ACCP) guidelines recommending anticoagulation for 3 months or until resolution of the precipitating risk factor for secondary PTE and longer anticoagulation of 6-12 months for idiopathic PTE.9) You et al. ${ }^{5)}$ reported a case in which extracorporeal membrane oxygenation (ECMO) with anticoagulation was helpful for a life-threatening condition caused by massive PTE in children with nephrotic syndrome. Massive PTE can cause hemodynamic instability, right ventricular failure, and circulatory collapse. ${ }^{8)}$ According to the 2016 ACCP Antithrombotic Guidelines, therapy for massive PTE should include systemic thrombolytic therapy combined with anticoagulation and supportive care.9) Systemic thrombolysis generally requires an intravenous infusion of 50-100 mg tissue plas-minogen activator over 1-2 hours and carries a 20\% risk of major bleeding and 2\%-5\% risk of hemorrhagic stroke. ${ }^{11)}$ However, in patients in whom systemic thrombolytics are contraindicated, ECMO and/or surgical embolectomy may be used to improve oxygenation, achieve hemodynamic stability, and successfully treat massive PTE. ${ }^{8)}$ The European Society of Cardiology 2014 guidelines state that ECMO can be used for massive PTE as a method of hemodynamic support and adjunct to surgical embolectomy. ${ }^{12)}$ Additionally, ECMO can be applied in the setting of massive PTE and hemodynamic instability, when invasive diagnostic studies are not feasible and urgent intervention is warranted.7)

In conclusion, the prevalence of PTE is increasing in children, and physicians' suspicion is important for prompt diagnosis, especially if there is an underlying cause such as nephrotic syndrome. ${ }^{13)}$ The rapid initiation of anticoagulation is important for favorable outcomes, and ECMO may be helpful when the patient is hemodynamically unstable. 


\section{Footnotes}

Conflict of interest: No potential conflicts of interest relevant to this article are reported.

See the article "Extracorporeal membrane oxygenation with systemic heparinization as a rescue therapy for acute lifethreatening pulmonary thromboembolism complicating nephrotic syndrome" via https://doi.org/10.3345/cep.2020. 01550 .

\section{References}

1. Leslom AN, Alrawiah ZMS, Al-Asmari AMA, Alqashaneen MDA, Alahmari AOT, Al-Ahmari HOBT. Prevalence of pulmonary thromboembolism in nephrotic syndrome patients: a systematic review and metaanalysis. J Family Med Prim Care 2020;9:497-501.

2. Barbano B, Gigante A, Amoroso A, Cianci R. Thrombosis in nephrotic syndrome. Semin Thromb Hemost 2013;39:469-76.

3. Gigante A, Barbano B, Sardo L, Martina P, Gasperini ML, Labbadia R, et al. Hypercoagulability and nephrotic syndrome. Curr Vasc Pharmacol 2014;12:512-7.

4. Mehls O, Andrassy K, Koderisch J, Herzog U, Ritz E. Hemostasis and thromboembolism in children with nephrotic syndrome: differences from adults. J Pediatr 1987;110:862-7.

5. You K, Kim SM, Kim D, Ryu K, Lee MJ. Extracorporeal membrane oxygenation with systemic heparinization as a rescue therapy for acute life-threatening pulmonary thromboembolism complicating nephrotic syndrome. Clin Exp Pediatr 2020 Nov 16 [Epub]. https://doi.org/ 10.3345/cep.2020.01550.

6. Rajpurkar M, Warrier I, Chitlur M, Sabo C, Frey MJ, Hollon W, et al. Pulmonary embolism-experience at a single children's hospital. Thromb Res 2007;119:699-703.

7. Yang Y, Lv J, Zhou F, Chen M, Wang R, Zhao M, et al. Risk factors of pulmonary thrombosis/embolism in nephrotic syndrome. Am J Med Sci 2014;348:394-8.

8. Ramiz S, Rajpurkar M. Pulmonary embolism in children. Pediatr Clin North Am 2018;65:495-507.

9. Monagle P, Chan AKC, Goldenberg NA, Ichord RN, Journeycake JM, Nowak-Göttl U, et al. Antithrombotic therapy in neonates and children: antithrombotic therapy and prevention of thrombosis, 9th ed: American College of Chest physicians evidence-based clinical practice guidelines. Chest 2012;141:e737S-e801S.

10. Radulescu VC. Anticoagulation therapy in children. Semin Thromb Hemost 2017;43:877-85.

11. Piazza G, Hohlfelder B, Jaff MR, Ouriel K, Engelhardt TC, Sterling KM, et al. A prospective, single-arm, multicenter trial of ultrasound-facilitated, catheter-directed, low-dose fibrinolysis for acute massive and submassive pulmonary embolism: the SEATTLE II study. JACC Cardiovasc Interv 2015;8:1382-92.

12. Weinberg A, Tapson VF, Ramzy D. Massive pulmonary embolism: extracorporeal membrane oxygenation and surgical pulmonary embolectomy. Semin Respir Crit Care Med 2017;38:66-72.

13. Carpenter SL, Richardson T, Hall M. Increasing rate of pulmonary embolism diagnosed in hospitalized children in the United States from 2001 to 2014. Blood Adv 2018;2:1403-8.

How to cite this article: Cho H. Pulmonary thromboembolism: a rare but life-threatening complication of nephrotic syndrome. Clin Exp Pediatr 2021;64:406-7. https://doi.org/ 10.3345/cep.2020.01942 